\title{
The Adoption of Mobile Health Applications Among University Students in Health Colleges
}

\author{
Abdulrahman M Jabour (D) \\ Wajiha Rehman' \\ Sumaira Idrees' \\ Hemalatha Thanganadar' \\ Kiani Hira ${ }^{2}$ \\ Mohammad A Alarifi ${ }^{3}$ \\ 'Department of Health Informatics, \\ Faculty of Public Health and Tropical \\ Medicine, Jazan University, Jazan, Saudi \\ Arabia; ${ }^{2}$ Maternal, Neonatal and Child \\ Health Research Network, Islamabad, \\ Pakistan; ${ }^{3}$ Department of Radiological \\ Sciences, College of Applied Medical \\ Sciences, King Saud University, Riyadh, \\ Saudi Arabia
}

Background: Studies documented the important role of health-care professionals in encouraging their patients to adopt and use mobile health applications. Health-care professionals who use and believe in mobile health applications are more likely to encourage and empower their patients to use it. Likewise, students in health-profession related specialties who use mobile health applications are more likely to continue to do so when they join the workforce.

Objective: The objective of this study was to examine the rate of adoption of mobile health applications in students in health colleges, identify the types of health applications used by health students, and identify the barriers that prevent the use of mobile health applications.

Methods: A descriptive cross-sectional study was conducted among university students. Students were invited to participate in a self-administrated survey from five health colleges. The survey included questions about the pattern of utilization, the purpose of the healthrelated application being used, and the barriers to adoption. The analysis included descriptive statistics using SPSS.

Results: A total of 383 students participated in this study. The within gender comparison showed that more female students were using health-related applications $59.8 \%$ compared to male students $49 \%$. The most frequent purpose of using mobile health applications were tracking physical activities $72.5 \%$ followed by counting calories intake $44 \%$. We also identified the frequency and duration of use as well as the factors which may impact students intention to use.

Conclusion: Fitness and dietary related applications were more common which might be explained by the fact that we only included a younger age group. The reported levels of perceived usefulness, accessibility and ease of use suggest that students will continue to use it.

Keywords: mHealth, mobile phones, behavior change, health promotion, physical activity, nutrition, overweight, adolescents and students

\section{Introduction}

There is an increasing interest in using mobile health application to advance the delivery of care. ${ }^{1,2}$ With the increasing use of mobile devices and internet availability, mobile health applications have become more accessible. ${ }^{3,4}$ The number of healthrelated applications in the Apple and Google Play stores exceeded 97,000 with approximately 1000 new applications being added per month. ${ }^{5}$ The value for mobile health adoption has become more apparent with the COVID-19 pandemic. ${ }^{6}$ Among the many factors which could impact the adoption of mobile health is the user's readiness and willingness to use it. ${ }^{7}$

While the smartphone applications can be promising for managing many of the chronic health conditions, studies repeatedly reported barriers related to its adoption
Correspondence: Abdulrahman M Jabour Department of Health Informatics, Faculty of Public Health and Tropical Medicine, Jazan University, Jazan, Saudi Arabia

Tel +966-17329500 Ext 5545

Email ajabour@jazanu.edu.sa 
and use. ${ }^{3,8}$ Despite the increase access to Internet and smartphones, studies repeatedly published seeking the understanding of users' adoption and the type of applications or functions they will be interested in., ${ }^{9,10}$

To advance beyond the generic recommendations seeking the understanding users' adoption, researchers focused on investigating the adoption of a particular type of users and age groups. ${ }^{8,11-15}$ For example, the digital divide has been reported as a major barrier to the adoption of diabetesrelated applications. ${ }^{13}$ As diabetes is more common among older patients, the digital divide may not apply to the younger generation. Therefore, understanding the pattern and use of each user group is essential for enhancing users' engagements and the design of user-centered application. ${ }^{11}$

To incorporate the mobile health applications in the delivery of care, researchers highlighted the importance of healthcare professionals' acceptance of mobile health. ${ }^{3,16,17}$ Studies reported the important role of health-care professionals in sustaining the patients' use and adoption of mobile health applications. ${ }^{18}$ Health-care professionals are more likely to promote and encourage their patients to use mobile health if they use it themselves or have a positive attitude toward it. Studies that examined the adoption of information technologies in general or mobile health have shown that perceived usefulness, ease of use, and accessibility are strong predictors of adoption and intention to use. ${ }^{22-24}$ Those studies uncovered many facilitators and barriers to adoption of mobile health applications among health-care professionals. ${ }^{3,9}$ Studies also investigated the use and attitude of mobile health apps among medical students as they are the future health-care professionals. ${ }^{14,15,19-21}$ While most of prior studies focus on medical students, little is known about students in other healthcare specialties such as nurses, dentists, and health education and promotion professionals.

Studies that investigated the use of health-related applications among students have inconsistent results. In the UK, $79.8 \%$ of students stated that they had a health-related application and that IOS users were more likely to use these applications compared to users of other operating systems. ${ }^{21}$ Another study conducted on university students at the University of Bordeaux in France found that only $34.9 \%$ of respondents had downloaded health-related applications. ${ }^{25}$ In the US, the cross-sectional survey found that $58 \%$ of the mobile phone owners had this type of application and that fitness and caloric intake applications were the most commonly downloaded. ${ }^{26}$ A study conducted in Greece found that $57.7 \%$ of medical students had one to five medical applications on their mobile devices. ${ }^{14}$ In Saudi Arabia, the regular use of health-related mobile applications was uncommon. Of those surveyed, $89.1 \%$ of users reported they had a healthrelated application. Approximately $73 \%$ were occasional users of this type of application and only $27 \%$ reported using this type of application at least once a day. ${ }^{27}$

To advance our understanding of the future health-care professional use and adoption of mobile health, we will focus on university students in health colleges. The objectives for our study are as follows:

- To identify the rate of adoption of mobile health applications in students in health colleges.

- To identify the types of health applications used by health students.

- To identify the barriers that prevent the use of mobile health applications.

\section{Methodology \\ Survey Developments}

We searched the literature for relevant survey tools and none of the existing surveys precisely met our needs. Therefore, we developed our own survey tools by adopting and modifying tools used in prior studies. ${ }^{3,9,20,21,26}$ The survey collected demographic information of students including gender, age, and level of study. The survey also included questions about the following: pattern of use, name of application, type of application being used, preferences about the applications, barriers, and facilitators (Appendix 1; Table S1).

A group of local experts (one statistician, two health informatics, and two medical doctors) had assessed the face and content validity of the questionnaire we developed using the Lawshe validation method. ${ }^{28,29}$ During this process, some of the questions were restructured and reorganized to improve the content validity of the questionnaire. To assess the reliability of the questionnaire, we used Kappa measure of agreement and Cronbach's alpha. We found significant and moderate results of Kappa and Cronbach's alpha which were greater than 0.5 indicating reliability and consistency in the questionnaire.

\section{Data Collection and Analysis}

This study was conducted on health college students at Jazan University in Saudi Arabia. After receiving Institutional Review Board approval (reference number REC41/4/082), students were invited to participate in the 
self-administrated survey. The study participants included male and female students in the Public Health, Nursing, Pharmacy, Applied Medical and Medical College. Students were recruited via convenience sampling by inviting students to participate during break hours. The study was conducted in accordance with the Declaration of Helsinki and participants were informed of the study details and were asked if they consent to participate in the study. A written consent was also provided along with the survey. The targeted a sample size was 368 at $95 \% \mathrm{CI}$. The questionnaire was translated into Arabic and was distributed on paper. The data were later transferred into Microsoft Excel for analysis. The analysis was performed using Microsoft Excel and Statistical Package for the Social Sciences SPSS V.21. The analysis includes descriptive analysis, Mann-Whitney, and chi squared tests.

\section{Results}

A total of 383 students participated in this study. All participants were undergraduate college students and the majority of participants were 20 years old or less, more than half $66 \%$ were females and the remaining 34\% were males. All study participants owned a smartphone. The overall percentage of students who currently own a healthrelated application was 56\% $(n=215 / 383)$. The gender distribution of those who currently own healthrelated applications was $49 \%$ for males and $59.8 \%$ for females.

The results showed that $(81 \%, n=311 / 383)$ of respondents own an iPhone and the remaining $(19 \%, n=72 / 383)$ using other operating systems including Android. Most study participants who owned a health-related application had three applications or less $(80 \%, n=173 / 215)$. Very few participants reported owning more than three applications.

The reasons for downloading the health applications are presented in Figure 1. When students were asked about the specific applications they own, the majority of the applications were in the fitness and exercise category (Appendix 2; Table S2). Students were also asked about the usefulness of their applications. Our results indicated that $71 \%$ of the respondents agreed that mobile applications reduced medical expenses and personal health management. Of the remaining respondents, 23\% were unsure if the applications reduced their expenses and eased health management and 3\% did not agree with this statement. No significant different was found between male and female in perceived usefulness $(P=0.355)$. We also calculated the chi-squared test to compare the difference in students' rating of the perceived usefulness across those who use mobile applications for, physical activities, counting calories, and losing weight, managing chronic disease, menstrual tracking and none of them showed significant difference with $P=0.987, P=0.285, P=0.555, P=0.810$, and $P=0.440$ respectively.

Mobile application usage was explored in several questions. The duration of application usage varied. Our study found that $28 \%$ used the application for six months, $18 \%$ used the application for 7 to 12 months, $20.6 \%$ used the application for one to two years, and $17 \%$ adopted the application for more than two years. We also found that $72.3 \%$ of students used the application at least twice a week (Table 1). The Mann-Whitney test showed that students who use mobile applications to count calories or weight loss reported a significantly higher frequency and duration of use compared to the other purposes such as tracking physical activities or recording menstrual cycle (Table 2).

For potential features that students found useful, 47\% $(n=102)$ selected "ease of use", $36 \%(n=77)$ selected "upto-date information", and $3 \%(n=6)$ selected "easily accessible". When participants were asked if they have ever paid for a mobile application, only 11\% answered "yes" and about half of the individuals who gave this answer said that they paid less than 50 Saudi riyals (13 USD) for the application. The results showed that Arabic was the preferred language for the majority of participants $(62 \%$; $\mathrm{n}=134)$, followed by English $(32 \%, \mathrm{n}=69)$, and both language $(6 \%, n=12)$. The most common reason for not using the application on a consistent basis was a lack of time $(57.6 \%, \mathrm{n}=124)$ followed by "difficult to use" $(23.2 \%$, $\mathrm{n}=50$ ) (Table 3).

Participants who did not download health applications were presented with statements that inquired why they did not download the applications. Of those who did not download the applications (168/383, 43\%), the top three reasons for not downloading were "not interested in health apps" (78/168, 46\%), followed by "My health is good and I do not need a health app" (34/168,20\%), and "complexity of use" $(21 / 168,12 \%)$.

\section{Discussion}

In this study we examined the adoption of mobile health applications among university students. We also examined the type of mobile health application which students use the most, as well as the barriers which may prevent them from using health-related applications. 


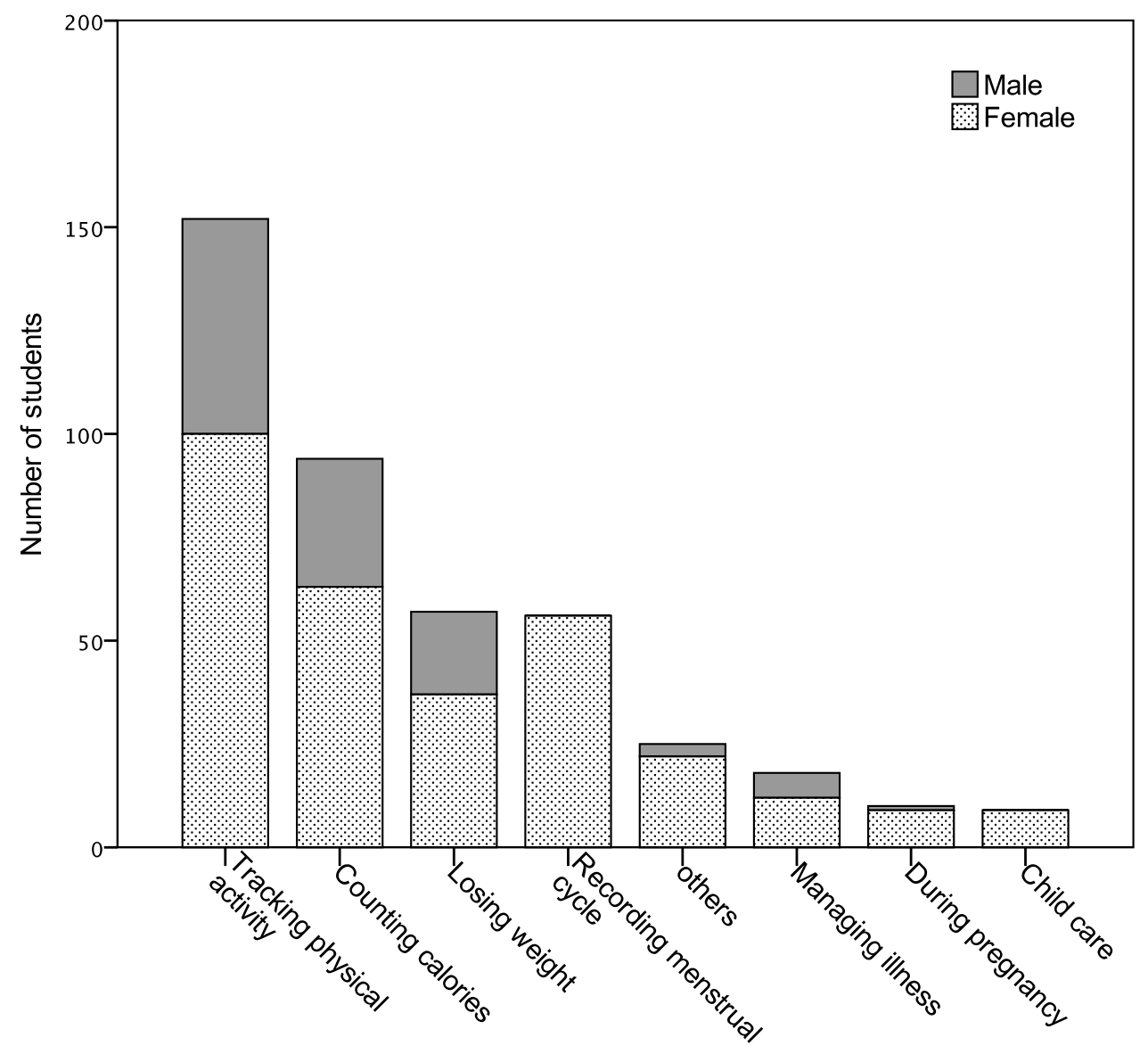

Figure I The reason for downloading health-related applications among university students.

With the rising rate of chronic diseases such as obesity and diabetes, many studies reported the important role of mobile health applications in disease management and prevention. Despite the rapid evolvement and technological advancement in mobile health applications, user's resistance and low level of utilization remain an issue. ${ }^{8,10,13}$ Health-care professionals have a very important role in promoting and encouraging patients to use mobile health applications. ${ }^{18}$ Health care

Table I The Frequency of Health-related Applications Use Among University Students

\begin{tabular}{|l|l|}
\hline Question Response & Participants n (\%) \\
\hline Several times a day & $38(17.6)$ \\
Once or twice a day & $64(29.7)$ \\
Two to three times a week & $54(25)$ \\
Once a week & $25(11.6)$ \\
Rarely used & $27(12.5)$ \\
Never used & $4(1.8)$ \\
Missing & $3(1.4)$ \\
Total & $215(100)$ \\
\hline
\end{tabular}

professionals who use a mobile health application and perceive it positively are more likely to promote mobile health applications to their patients. Understanding the adoption of mobile health applications among the students in health-related colleges is equally important as they are the future health-care workforce.

Both perceived usefulness, ease of use, and accessibility are a strong predictor of user's intention to use and adoption of information technologies. ${ }^{22-24}$ Our study found that $71 \%$ users agreed that health application can aid in reducing health related cost and improve their health. We also did not find any difference in the perceived usefulness between gender or between the groups using mobile applications for the different purposes such as physical activities, counting calories, and losing weight, managing chronic disease, or mentstrual tracking. We also found that all respondents had access to smartphones, which reflects the high potential of smartphone technologies in accessing this age group. Moreover, our results show that many of the digital divides and technology access barriers reported in prior studies are not applicable to this age group. Another important predictor for user's 
Table 2 Comparing the Difference in the Frequency of Using the Applications and Duration Users Spent Daily Using the Applications by the Different Purpose of Use

\begin{tabular}{|l|l|l|l|l|l|}
\hline \multirow{2}{*}{ Purpose of Use } & \multicolumn{2}{l|}{ Frequency of Using the Applications } & \multicolumn{2}{l|}{ Duration Users Spent Daily Using the Applications } & $\mathbf{N}^{\mathbf{a}}$ \\
\cline { 2 - 5 } & P-value & Median & P-value & Median \\
\hline Track physical activities & 0.190 & Once or twice a day & 0.086 & $\mathrm{I}-10 \mathrm{~min}$ & $\mathrm{I}$ \\
Count calories & $0.00 \mathrm{I}$ & Once or twice a day & 0.003 & $\mathrm{II}-20 \mathrm{~min}$ & 86 \\
Weight loss & $0.01 \mathrm{I}$ & Once or twice a day & 0.007 & $\mathrm{II}-20 \mathrm{~min}$ & 50 \\
Recording menstrual cycle & 0.595 & Two to three times a week & 0.219 & $\mathrm{I}-10 \mathrm{~min}$ \\
\hline
\end{tabular}

Notes: ${ }^{a} \mathrm{~N}$ out of the 198 who responded yes to owning a health-related application.

Table 3 University Students Response to "Reasons to Not Use Downloaded Applications on a Consistent Basis"

\begin{tabular}{|l|l|}
\hline Question Response & Participants n (\%) \\
\hline Shortage of time & $124(57.6)$ \\
Difficult to use & $50(23.2)$ \\
Not useful & $3(1.4)$ \\
Language & $16(7.5)$ \\
Do not know how to use it & $17(7.9)$ \\
Missing & $5(2.3)$ \\
Total & $215(100)$ \\
\hline
\end{tabular}

intention to use and adoption of information technologies is the ease of use. ${ }^{24}$ We found that only $12 \%$ of those who do not own a health-related application reported the reason for not using it was complexity of use.

Despite the high number of smartphone users, we found that little over half $(56 \%)$ of students had downloaded a healthrelated application. This result was consistent with other studies conducted in the US (58\%) and Greece $(57.7 \%)$ on this topic. ${ }^{14,26}$ Other studies have reported a different proportion of health-related application owners among students ranging from $34.9 \%$ in France to $79.8 \%$ in the UK. ${ }^{21,25}$ Despite the similarity in age and level of education in the people studied, there was a noticeable difference in the level of adoption.

To identify the type of health-related application owned by students, we asked them to select the category which describes the purpose of the application as well as naming the specific application they would recommend. We found the majority of respondents used applications to track physical activity, weight loss, calorie counting, and menstrual cycle tracking. The most used applications were MyFitnessPal, Stepz, Sehati, Fitbit, and Hayat. While many of the published articles about mobile health applications discussed the importance of these tools in managing chronic illnesses like diabetes and hypertension, our results showed that only $8 \%$ to $9 \%$ of students who used these types of applications were using the applications for this purpose. An explanation for this low usage among our population is due to the age of our study ( $<20$ years). This group could, generally, be more interested in monitoring things that were not chronic conditions and could find greater use in tracking calories, losing weight, and keeping a record of physical activity. ${ }^{13}$ The high proportion of health application owners who use their mobile for physical activity $(72.5 \%)$ or counting caloric intake (44\%) suggests that it would be far more practical to utilize mobile applications to target the preventative and healthy lifestyle goals than disease management for this age group.

In addition, characterizing health applications with physical activity and counting caloric intake may explain why $43 \%$ of respondents did not download any type of health application at all. The most commonly selected statements were "not interested in health apps" and "My health is good and I do not need a health app". We were unable to determine if $43 \%$ of respondents who did not download any type of health application perceived health applications as a disease management application that only applied to people with a specific disease or applications that could promote a healthy lifestyle and applies to anyone. More information about their health status, selfefficacy, and goals might be incorporated in future studies.

An interesting finding was that the cost of the application did not seem to be a barrier to application use. Only $11 \%$ of users reported paying for an application, but this could be due to the many free applications available in the marketplace.

One limitation of our study is that we only included students of the health colleges which may not be representative of the whole university or users of the same age group. Another limitation was that barriers and factors reported by students were limited to the options available in our questionnaire. In future studies, we recommend conducting focus groups and propping questions. This will provide a deeper understanding of the factors which may influence the adoption and use of mobile health applications. 
One recommendation when designing for universityage students is to place more emphasis on promotion and fitness rather than managing chronic diseases. This will also guide the design principles in each type of application to suit the targeted age group. For example, apps for managing chronic disease would need to focus on the simplicity and ease of use to be accessible for older generations while fitness and calorie tracker related apps could include more functions to meet the need of younger users.

Another recommendation for future studies is to leverage the power of the different health-care professionals in promoting the adoption and use of mobile health. While the focus has been on physicians and nurses, we recommended exploring ways of how other health-care professionals like health education professionals, nutritionists, pharmacists, and public health specialists can participate in promoting and encouraging the adoption of mobile health applications related to their specific field.

\section{Conclusion}

This study provided insight into the adoption rate of health-related applications among university students in health colleges. The majority of applications adopted by students were related to fitness and calorie counting. As students adopt mobile health applications at a younger age and find them useful, they are more likely to continue using mobile health related applications and promote them in the future. Promoting the adoption of these types of applications among younger individuals on a large scale can be a great preventative health strategy since obesity and diabetes is a global public health burden. Investing in applications to target the younger demographic can have advantages over traditional approaches since applications can be scaled to larger audiences at no extra cost and the applications could be created in a way to monitor user data and measure the effectiveness of interventions. One of the limitations of our study is that it was conducted in a single university. For future studies, we recommend expanding the study to a larger population to examine the generalizability of our results. We also recommend adding focus groups for deeper understanding of the student's perceptions and the factors which may influence their level of adoption.

\section{Acknowledgments}

The authors would like to thank all students who participated in this survey.

\section{Author Contributions}

All authors have contributed to the study designs, drafting and editing. The primary author AJ has supervised the project design, execution and performed the analysis. The second author WR has supervised the process of data collection, and management of participants. All authors critically reviewed, edited, and approved the final manuscript.

\section{Funding}

There are no funding resources to declare.

\section{Disclosure}

The authors reported no conflicts of interest in this work and have no competing financial, professional, or personal interests that might have influenced the work described in this manuscript.

\section{References}

1. Free C, Phillips G, Watson L, et al. The effectiveness of mobile-health technologies to improve health care service delivery processes: a systematic review and meta-analysis. PLoS Med. 2013;10(1):e1001363. doi:10.1371/journal.pmed.1001363

2. Marcolino MS, Oliveira JAQ, D'Agostino M, Ribeiro AL, Alkmim MBM, Novillo-Ortiz D. The impact of mhealth interventions: systematic review of systematic reviews. JMIR Mhealth Uhealth. 2018;6(1):e23. doi:10.2196/mhealth.8873

3. Gagnon M-P, Ngangue P, Payne-Gagnon J, Desmartis M. m-Health adoption by healthcare professionals: a systematic review. $J$ Am Med Inform Assoc. 2015;23(1):212-220. doi:10.1093/jamia/ocv052

4. Ricciardi L, Mostashari F, Murphy J, Daniel JG, Siminerio EP. A national action plan to support consumer engagement via e-health. Health Aff. 2013;32(2):376-384. doi:10.1377/hlthaff.2012.1216

5. Peng W, Kanthawala S, Yuan S, Hussain SA. A qualitative study of user perceptions of mobile health apps. BMC Public Health. 2016;16. doi:10.1186/s12889-016-3808-0

6. Bokolo AJ. Application of telemedicine and eHealth technology for clinical services in response to COVID-19 pandemic. Health Technol (Berl). 2021;11(2):359-366. doi:10.1007/s12553-020-00516-4

7. Anthony B. Implications of telehealth and digital care solutions during COVID-19 pandemic: a qualitative literature review. Inform Health Soc Care. 2021;46(1):68-83. doi:10.1080/17538157.2020.1839467

8. LeRouge C, Van Slyke C, Seale D, Wright K. Baby boomers' adoption of consumer health technologies: survey on readiness and barriers. J Med Internet Res. 2014;16(9):e200. doi:10.2196/jmir.3049

9. Kwon M, Mun K, D'Angelo J, McLeod DM, Lee J. Integrating mobile communication technologies into public health: identifying the motivational factors of health app engagement. International Communication Association 65th Annual Conference, Caribe Hilton, San Juan, Puerto Rico; 21 May 2015; Puerto Rico.

10. Fahad Aldhaban TUD, Harmon R. Exploring the adoption and use of the smartphone technology in emerging regions. Paper presented at: Management of Engineering and Technology (PICMET) 2015 Portland International Conference on; 2016; 2355-2370; Portland.

11. Wang Y, Min J, Khuri J, et al. Effectiveness of mobile health interventions on diabetes and obesity treatment and management: systematic review of systematic reviews. JMIR Mhealth Uhealth. 2020;8 (4):e15400. doi:10.2196/15400 
12. Winter SJ, Sheats JL, King AC. The use of behavior change techniques and theory in technologies for cardiovascular disease prevention and treatment in adults: a comprehensive review. Prog Cardiovasc Dis. 2016;58(6):605-612. doi:10.1016/j.pcad.2016.02.005

13. Jabour A, Jones JF. Facilitators and barriers to patients' engagements with personal health records: systematic review. Paper presented at: International Conference on Universal Access in Human-Computer Interaction; 2013.

14. Chatzipavlou I, Misirlis N, Vlachopoulou M. Smartphone medical app use: a survey among medical students at Aristotle University of Thessaloniki. MCIS 2015Proc. 2015.

15. Cho J, Quinlan MM, Park D, Noh GY. Determinants of adoption of smartphone health apps among college students. Am J Health Behav. 2014;38:860-870. doi:10.5993/AJHB.38.6.8

16. Leigh S, Ashall-Payne L, Andrews T. Barriers and facilitators to the adoption of mobile health among health care professionals from the United Kingdom: discrete choice experiment. JMIR Mhealth Uhealth. 2020;8(7):e17704. doi:10.2196/17704

17. Gagnon M-P, Desmartis M, Labrecque M, et al. Systematic review of factors influencing the adoption of information and communication technologies by healthcare professionals. J Med Syst. 2012;36 (1):241-277. doi:10.1007/s10916-010-9473-4

18. Miyamoto SW, Henderson S, Young HM, Pande A, Han JJ. Tracking health data is not enough: a qualitative exploration of the role of healthcare partnerships and mHealth technology to promote physical activity and to sustain behavior change. JMIR mHealth uHealth. 2016;4(1):e5. doi:10.2196/mhealth.4814

19. Jebraeily M, Fazlollahi ZZ, Rahimi B. The most common smartphone applications used by medical students and barriers of using them. Acta Informatica Medica. 2017;25(4):232-235. doi:10.5455/ aim.2017.25.232-235

20. Quant C, Altieri L, Torres J, Craft N. The self-perception and usage of medical apps amongst medical students in the United States: a cross-sectional survey. Int J Telemed Appl. 2016;2016:5. doi:10.1155/ 2016/3929741
21. Payne KB, Wharrad H, Watts K. Smartphone and medical related app use among medical students and junior doctors in the United Kingdom (UK): a regional survey. BMC Med Informat Decis Making. 2012;12. doi:10.1186/1472-6947-12-121

22. Garavand A, Samadbeik M, Nadri H, Rahimi B, Asadi H. Effective factors in adoption of mobile health applications between medical sciences students using the UTAUT model. Methods Inf Med. 2019;58(04/05):131-139. doi:10.1055/s-0040-1701607

23. Mittal A, Aggarwal A, Mittal R. Predicting university students' adoption of mobile news applications: the role of perceived hedonic value and news motivation. Int J E-Serv Mobile Appl. 2020;12 (4):42-59. doi:10.4018/IJESMA.2020100103

24. Larcker DF, Lessig VP. Perceived usefulness of information: a psychometric examination. Decis Sci. 1980;11(1):121-134. doi:10.1111/j.1540-5915.1980.tb01130.x

25. Montagni I, Cariou T, Feuillet T, Langlois E, Tzourio C. Exploring digital health use and opinions of university students: field survey study. JMIR mHealth uHealth. 2018;6(3):e65. doi:10.2196/ mhealth.9131

26. Krebs P, Duncan DT. Health app use among us mobile phone owners: a national survey. JMIR mHealth uHealth. 2015;3:e101. doi:10.2196/ mhealth.4924

27. Sayedalamin Z, Alshuaibi A, Almutairi O, Baghaffar M, Jameel T, Baig M. Utilization of smart phones related medical applications among medical students at King Abdulaziz University, Jeddah: a cross-sectional study. J Infect Public Health. 2016;9:691-697. doi:10.1016/j.jiph.2016.08.006

28. Lawshe CH. A quantitative approach to content validity. Pers Psychol. 1975;28(4):563-575. doi:10.1111/j.1744-6570.1975. tb01393.x

29. Taherdoost H. Validity and reliability of the research instrument; how to test the validation of a questionnaire/survey in a research. How to test the validation of a questionnaire/survey in a research (August 10, 2016). 2016
Journal of Multidisciplinary Healthcare

\section{Publish your work in this journal}

The Journal of Multidisciplinary Healthcare is an international, peerreviewed open-access journal that aims to represent and publish research in healthcare areas delivered by practitioners of different disciplines. This includes studies and reviews conducted by multidisciplinary teams as well as research which evaluates the results or conduct of such teams or healthcare processes in general. The journal covers a very wide range of areas and welcomes submissions from practitioners at all levels, from all over the world. The manuscript management system is completely online and includes a very quick and fair peer-review system. Visit http://www.dovepress.com/testimonials. php to read real quotes from published authors. 